\title{
HERITAGE AND TERRITORY PROJECT: CASE OF RABAT (MOROCCO)
}

\author{
Bouchra Laamrani*, ORCID ID: 0000-0003-3407-609X, \\ Mounir Zouiten, ORCID ID: 0000-0001-7742-191X \\ ${ }^{1}$ Mohammed V. University, United Nations Avenue, Agdal, Rabat, Morocco \\ *Corresponding author: Bouchra Laamrani, bouchra.laamrani@um5s.net.ma
}

Received: 07. 16. 2021

Accepted: 08. 28. 2021

\begin{abstract}
To counterbalance the stakes of globalization and generalized competition, territories are compelled to build and manage their territorial attractiveness which is, often, driven by a comparative or even a differentiating advantage. Aware of these issues, the actors of the city of Rabat have undertaken territorialized actions. Indeed, the built fabric of this city is included in the UNESCO World Heritage List. In addition, the city has adopted an integrated urban development program. This article aims to examine the reconciliation between the heritage status of the city and the urban projects undertaken within the framework of this program. The analysis is based on a documentary study.
\end{abstract}

Keywords: actors, cultural heritage, Rabat, urban project, world heritage.

Rezumat. Pentru a contrabalansa miza globalizării și a concurenței generalizate, teritoriile sunt obligate să-și construiască și să-și gestioneze atractivitatea teritorială, care este, adesea, determinată de un avantaj comparativ sau chiar diferențiat. Conștienți de aceste probleme, actorii orașului Rabat au întreprins acțiuni teritorializate. Într-adevăr, structura de construcții a acestui oraș este inclusă pe lista patrimoniului mondial UNESCO. În plus, orașul a adoptat un program integrat de dezvoltare urbană. Acest articol își propune să examineze reconcilierea dintre statutul de patrimoniu al orașului și proiectele urbane întreprinse în cadrul acestui program. Analiza se bazează pe un studiu documentar.

Cuvinte cheie: actori, patrimoniu cultural, Rabat, proiect urban, patrimoniu mondial.

\section{Introduction}

L'attractivité d'un territoire est souvent rapportée à sa faculté de capter et à retenir des ressources mobiles, notamment les facteurs de production et la population. Elle se définit, au sens de Poirot et Gérardin, comme « la capacité d'un territoire à être choisi par un acteur comme zone de localisation (temporaire ou durable) pour tout ou partie de ses activités » [1].

En raison des menaces grandissantes émergées d'un contexte mondial perturbé, les territoires se donnent tous les moyens pour bâtir leurs attractivités territoriales et la maintenir. De nouvelles pratiques se développent alors, en l'occurrence le marketing territorial ou encore le branding territorial, d'autres s'éclipsent totalement ou partiellement. La notion de "plan", longtemps adoptée dans le cadre de l'aménagement et de l'urbanisme, est supplantée par la notion de "projet urbain". Donner un nouvel élan à son attractivité, le projet de territoire s'entend comme un nouveau type d'actions impliquant de plus en plus les collectivités territoriales [2]. 
Dans une autre perspective, développer l'attractivité d'un territoire repose sur la valorisation de ses ressources spécifiques, territoriales, voire patrimoniales. En effet, la valorisation de ces ressources profère au territoire un avantage différenciatif, d'où l'engouement, ces dernières décennies, des inscriptions sur la liste du patrimoine mondial de l'UNESCO. Ayant la faculté de donner une reconnaissance mondiale aux biens inscrits, ces inscriptions présentent également des contraintes, notamment celles liés aux critères d'inscription.

En 2012, le patrimoine culturel bâti de la ville de Rabat a été inscrit sur la liste du patrimoine mondial de l'UNESCO, selon deux critères de classement (II) et (IV) [3]. En 2014, la capitale s'est dotée de son programme de développement urbain ayant pour objectif de développer le tissu urbain selon une vision cohérente et équilibrée.

Cet article tente, de savoir si les objectifs des projets urbains riment avec les contraintes de la patrimonialisation. Il analyse l'articulation entre la valorisation du patrimoine urbain (bâti) et les projets de territoire (urbain) de la ville de Rabat. Pour ce faire, la mobilisation des documents scientifiques et rapports produits par l'UNESCO, le ministère de la Culture et le ministère de l'Aménagement des territoires, nous a été d'une grande utilité.

\section{La patrimonialisation, un processus porté par les acteurs territoriaux}

\subsection{Bref aperçu sur la notion du patrimoine culturel}

La notion de patrimoine s'est imposée au tournant des années 1970 - 1980. Sa prolifération s'explique principalement par le post-fordisme, mais aussi par la crise globale de la modernité et les mutations sociales, notamment les mutations professionnelles, la crise identitaire et la mondialisation [4]. Se pencher sur le processus de valorisation du patrimoine renvoie à l'exercice de cerner sa définition dans un premier temps. C'est une notion qui se démarque par sa polysémie. Le patrimoine matérialise alors l'identité $[5,6]$, comme il constitue une ressource pour le développement local, voire territorial [7 - 10]. Il est également un levier du tourisme, notamment du tourisme culturel [11].

L'approche économique du patrimoine lui confère sa capacité d'engendrer des fonctions non marchandes, à côté de sa fonction marchande que lui reconnaît le marché. L'approche politique du patrimoine lui attribue la faculté de distinguer des groupes et de les relier à d'autres [12].

Pour ce qui concerne le patrimoine culturel, celui-ci se définit, selon Berriane et Michon (2016), comme « une construction sociale. Il n'existe pas, il advient à partir du moment où des individus ou des groupes en proclament l'existence ou le souhait d'existence » [13]. "Le patrimoine n'existe pas a priori » [14]. Le bien, selon Di Méo (2007), acquiert la qualité de patrimoine en lui appliquant le processus de patrimonialisation. Ce processus prend appui sur la mobilisation des acteurs qui revêt un caractère central [4].

\subsection{Le Processus de patrimonialisation}

La patrimonialisation, au sens d'Amougou (2004), est un processus social par lequel les acteurs légitimes confèrent à un objet, à un espace (architectural, urbanistique ou paysager) ou encore une pratique sociale, des "valeurs" reconnues et partagées, par les agents légitimés eux-mêmes pour les transmettre ensuite à l'ensemble des individus. Cette transmission se matérialise, pour l'essentiel, par des mécanismes d'institutionnalisation nécessaires à la préservation [15]. Ce processus est également compris en tant que construction sociale dans la mesure où "patrimonialiser" un bien, c'est lui conférer un "sens" [16]. Dans la même lancée, Davallon (2014) entend la patrimonialisation comme la 
reconnaissance d'un collectif du statut de patrimoine à des biens matériels et immatériels. Ce collectif se charge alors de préserver ce patrimoine et de le transmettre [17].

De manière tout aussi générale, dans la littérature, il est bien établi que la patrimonialisation et ses processus ne sont pas neutres. Elle repose sur la coordination des acteurs locaux, voire territoriaux. Les processus sont engagés dans la perspective de conférer à l'objet sa qualité de patrimoine, pour acquérir éventuellement, la qualité de ressource territoriale ou ressource patrimoniale. Ces ressources sont souvent engagées dans les projets de territoires ou encore dans les programmes de développement comme elles sont sollicitées lors de la conception des stratégies de marketing territorial.

Le processus de patrimonialisation est entrepris, certes, par les acteurs, mais il est souvent provoqué par un événement déclencheur. Le tourisme, de par la nature de ses activités, a la faculté de participer à la révélation de la ressource et de déboucher sur sa valorisation [18]. Le projet de territoire est qualifié par Courlet (2013) comme un processus de révélation des ressources [2].

\section{Projet de territoire}

\subsection{Un construit d'acteurs}

Le projet est l'un des instruments privilégiés de l'urbanisme pour transformer et organiser le fonctionnement de l'espace [19]. En effet, au tournant des années 1970, la notion de "plan" qui se veut un vecteur d'une domination du savoir expert, est supplantée par celle de "projet urbain" qui se présente par contre, comme un processus concerté, démocratique et indéterminé [20]. Il se situe comme un projet d'ouverture dans la mesure où il impose une évolution des mentalités [21]. Ce glissement renseigne sur le changement de la manière de penser l'action publique. Par le passé, celle-ci se réduisait à une opération de mise en œuvre d'un savoir technique universel. L'action publique, au prisme du projet repose, sur l'implication des acteurs du territoire et la valorisation de ses ressources [20].

La distinction est faite entre le "projet de développement", "territoire de projet" et " projet de territoire". Cette distinction est surtout relative à l'acception de la notion de territoire à caractère polysémique. Le "projet de développement" fait référence notamment à des projets conçus par les institutions internationales d'aide au développement; ils sont souvent accompagnés d'une structure de gestion [22]. Le "projet de territoire", quant à lui, repose sur la coordination des acteurs du territoire au travers la construction de modes de gouvernance adaptés. Il s'appuie sur le territoire selon son acception de "territoire construit" [23]. Dans cette perspective, le projet de territoire, selon Zouiten (2004), est conçu pour permettre aux acteurs locaux et à leurs institutions d'acquérir des capacités correspondant aux dimensions de la compétitivité territoriale. Il s'agit de la capacité à valoriser leur environnement, à agir ensemble, à établir des liens entre les départements, et in fine celle à établir des liens avec d'autres territoires [24]. Le "Territoire de projet" repose sur la notion de "territoire donné", s'appuyant sur la coordination des partenaires institutionnels extérieurs aux territoires [23]. Ces acteurs sont amenés à coopérer pour concevoir et participer à la réalisation d'un projet pour le territoire. Cependant, il demeure difficile de les amener à construire des modes de gouvernance durable [22].

\subsection{Une démarche de révélation des ressources}

Le projet est un instrument de mobilisation sociale [20]. Ses processus, prenant appui sur la coordination et l'implication des acteurs du territoire concerné, ont éventuellement la faculté d'actualiser, de produire et de valoriser des ressources territoriales $[2,25]$. Le projet 
urbain est diamétralement opposé au plan. Il cherche, au travers de ses démarches, « de renouer avec une urbanisation intensive ménageant la ville existante, de valoriser le patrimoine bâti et le patrimoine des pratiques sociales » [20], alors que le plan repose sur la vision de l'urbanisme comme "science des savoirs experts, de la prévision, d'un urbanisme de la table rase peu soucieux de la ville existante » [20]. Le projet de territoire questionne l'articulation entre le territoire et ses ressources [2].

Si nombre d'auteurs appréhendent le projet de territoire comme révélateur ou régulateur de la ressource, d'autres s'interrogent, par contre, sur l'accommodement entre ses objectifs et ceux de la patrimonialisation. Dans cette dernière optique, Greffe (2000) précise que «... protéger ce patrimoine urbain - ou mieux encore jusqu'à quel point il faut le faire - se situe au cour d'une démarche élargie [...] qui dépasse très largement celle de la conservation et doit d'ailleurs prendre en considération des stratégies directement opposées à celle de la conservation » [8]. En effet, le patrimoine étant une sorte de construction sociale, sa place dans les projets dépend indéniablement des objectifs de développement que se fixe la société concernée, mais aussi des autres types de ressources disponibles sur le territoire [26].

\section{Projets urbains de Rabat et sa patrimonialisation,}

En 2012, la ville de Rabat a fait inscrire son patrimoine culturel bâti sur la liste du patrimoine mondial de l'UNESCO. Cette inscription profère au tissu bâti de la capitale une reconnaissance internationale. Deux ans plus tard, la ville s'est dotée d'un programme intégré de développement, baptisé "Rabat, ville lumière et capitale culturelle du Maroc".

\subsection{L'inscription de Rabat sur la liste du patrimoine mondial UNESCO}

L'inscription de la ville de Rabat sur la liste du patrimoine mondial de l'UNESCO en 2012 repose sur de deux critères. Rabat est reconnue comme un témoignage d'un échange d'influences considérables. En effet, le chevauchement des éléments relevant du style marocain et européen dote la ville d'un caractère original et nouveau. La capitale est reconnue également comme exemple éminent d'un type de construction dans la mesure où elle incarne un modèle d'urbanisme distingué, intégrant des valeurs du passé dans le cadre de son projet de ville contemporaine. Il s'agit des critères de classement (II) et (IV) [3].

Dans le cadre de la conservation et la gestion du patrimoine bâti de la capitale, plusieurs projets ont été mis en place, dont l'objet, l'objectif et les budgets alloués sont détaillés dans le « tableau 1 » ci-dessous:

Tableau 1

Projets de restauration, d'aménagement et de promotion des médinas, des sites et des monuments historiques [27].

\begin{tabular}{|c|c|c|c|}
\hline Projet & Localisation & $\begin{array}{l}\text { Délai de } \\
\text { réalisation }\end{array}$ & $\begin{array}{l}\text { Coût estimé } \\
\text { en MAD }\end{array}$ \\
\hline $\begin{array}{l}\text { Aménagement et mise en valeur de la Kasbah des } \\
\text { Oudayas }\end{array}$ & Rabat & 02 ans & 50000000,00 \\
\hline $\begin{array}{l}\text { Restauration et aménagement du site } \\
\text { archéologique de Chellah }\end{array}$ & Rabat & 02 ans & 50000000,00 \\
\hline Restauration et aménagement de la Tour Hassan & Rabat & 02 ans & 18000000,00 \\
\hline $\begin{array}{l}\text { Restauration et aménagement des grottes de Dar } \\
\text { Es Soltane }\end{array}$ & Rabat & 02 ans & 3000000,00 \\
\hline & & & $\begin{array}{r}121000 \\
000,00 \\
\end{array}$ \\
\hline
\end{tabular}


Ces projets s'inscrivent dans la stratégie patrimoine 2020, qui s'articule autour de deux objectifs stratégiques. Ces objectifs se traduisent par la préservation et la valorisation du patrimoine culturel, d'une part, et le développement d'une économie du patrimoine en tant que pôle de création de la richesse, d'autre part [27].

Cette inscription confère une reconnaissance internationale au patrimoine immobilier de la ville. Une telle reconnaissance peut servir de pilier majeur dans le cadre de la conception de son marketing territorial, afin de renforcer son attractivité. Toutefois, elle stipule également des recommandations à respecter lors de la mise en place des projets urbains programmés pour la capitale.

\subsection{Le Programme intégré de développement urbain de la ville de Rabat}

En 2014, Rabat s'est doté de son Programme intégré de développement urbain, baptisé "Rabat ville lumière, Capitale Marocaine de la culture". Porté par l'Etat, ce programme s'articule autour de sept principaux axes. Il vise à valoriser le patrimoine culturel et civilisationnel, préserver les espaces verts et l'environnement, faciliter l'accès aux services et équipements sociaux de proximité tout en renforçant la gouvernance. Quant au pilotage opérationnel (maîtrise d'ouvrage) du programme, il est assuré par la société "Rabat, Région, Aménagement".

Les projets urbains, entrepris dans le cadre de ce programme de développement, ont pour objectif principal d'accroître les infrastructures économiques, sociales et culturelles de la ville selon une vision cohérente et équilibrée. Il comprend un nouveau théâtre, un nouveau musée national d'Archéologie et des Sciences, des parkings souterrains et des propositions de nouveaux paysages urbains dans la zone tampon, ainsi qu'un bâtiment d'une grande hauteur, la « O Tower » situé à l'extérieur de la zone tampon et l'extension de la gare.

Ces deux derniers projets - «O Tower» et l'extension de la gare - ont suscité les inquiétudes de l'UNESCO, notamment par rapport à l'intégrité visuelle du bien inscrit et de ses environs. Ces inquiétudes ont fait l'objet des recommandations adressées au Maroc en juillet 2019 [28].

\subsection{Projets urbains et enjeux du classement}

Le comité du patrimoine mondial s'inquiète par rapport à l'impact visuel négatif éventuel de la tour « O Tower » de 55 étages, sur toute la ville de Rabat surtout que la ville a un profil horizontal. La mission de conseil estime que ce projet a un impact très nuisible sur la tour Hassan, le mausolée du roi Mohamed $V$ et la Kasbah des Oudaïas, dans le sens où il réduit leur prédominance visuelle. La mission avait recommandé alors de reconsidérer l'emplacement de la tour [28].

La mission s'inquiétait également par rapport à l'extension de la gare ferroviaire qu'elle estime avoir un impact visuel négatif sur les murailles de la ville de par le fait qu'elle soit grande et dominante visuellement et physiquement. Le comité précise également qu'aucune étude d'impact n'a été réalisée pour évaluer l'impact visuel de ces projets afin de préserver l'intégrité visuelle de la ville [28].

Le Maroc, estime de son côté que la tour a été conçue de telle sorte à ce qu'elle soit vue comme un "miroir réfléchissant postmoderne" pour la ville et l'incarnation de sa modernisation. Il soutient sa position en précisant que la convention n'offre pas assez de directives quant à l'évaluation de l'impact des projets au-delà des zones tampons. Pour ce qui est de l'extension de la gare, le Maroc explique que l'état d'avancement des travaux de construction ne permet pas de réaliser de profondes modifications au niveau des plans [28]. 


\section{Conclusions}

La nécessité de protéger et de transmettre le patrimoine définit comme "bien commun" [29], renvoie à la mise en place d'un processus de patrimonialisation dont l'objectif est de restaurer et conserver un patrimoine donné, mais aussi de le valoriser. L'inscription du patrimoine bâti de la ville de Rabat se situe dans cette logique. Moderniser la ville, bâtir et développer son attractivité sont des activités tributaires de la cohérence de ses différents projets urbains reposant sur la coordination et l'implication de ses différents acteurs territoriaux.

Les recommandations adressées au Maroc par rapport à deux projets urbains laissent comprendre que l'inscription sur la liste du patrimoine mondiale n'épargne en rien le fait de rester attaché aux critères sur lesquels repose l'inscription.

Le cas de Porto au Portugal, dont le centre historique est déclaré comme patrimoine mondial en 1996 par l'UNESCO, et la mise en place de son projet urbain « Frente Ribeirinha », ou encore celui de Harar en Éthiopie, inscrite sur la liste en 2006, sont assez parlants quant à la conciliation de la patrimonialisation et des projets urbains.

\section{Références bibliographiques}

1. Poirot J., Gérardin H. L'attractivité des territoires: un concept multidimensionnel. Mondes en développement. 2010;149(1):27 - 41.

2. Courlet C., Kadiri NE., Fejjal A., Jennan L. Le projet de territoire comme construit d'acteurs et processus de révélation des ressources: l'exemple marocain. GéoDév.ma [Internet]. 2013;1(0). Disponible sur: https://revues.imist.ma/index.php?journal=GeoDev\&page=article \&op=view\&path\%5B\%5D=612

3. UNESCO. Comité du patrimoine mondial. Propositions d'inscription de biens sur la Liste du patrimoine mondial-2012 [Internet]. 2012. Disponible sur: https://whc.unesco.org/fr/documents/116721/

4. Di Méo G. Processus de patrimonialisation et construction des territoires. 12 sept 2007; Disponible sur: https://halshs.archives-ouvertes.fr/halshs-00281934

5. Di Méo G. Patrimoine et territoire, une parenté conceptuelle. Espaces et sociétés. 1994;(4):15-34.

6. Pelissier M., Pybourdin I. L'intelligence territoriale. Les Cahiers du numérique. 2009;Vol. 5(4):93-109.

7. Greffe X. La valeur économique du patrimoine [Internet]. Anthropos-Economica. Paris; 1990.

Disponible sur: https://www.decitre.fr/ebooks/la-valeur-economique-du-patrimoine9782402479134_9782402479134_1.html

8. Greffe X. Le patrimoine comme ressource de la ville. Les Annales de la recherche urbaine. 2000;(86): $29-38$.

9. Landel P-A., Senil N. Patrimoine et territoire, les nouvelles ressources du développement. Développement durable et territoires Économie, géographie, politique, droit, sociologie [Internet]. 2009; (Dossier 12). Disponible sur: http://journals.openedition.org/developpementdurable/7563

10. Cominelli F, Greffe X. L'économie politique du patrimoine culturel. In Situ Au regard des sciences sociales [Internet]. 15 oct 2019 ;(1). Disponible sur: http://journals.openedition.org/insituarss/436

11. Yu X., Xu H. Cultural heritage elements in tourism: A tier structure from a tripartite analytical framework. Journal of Destination Marketing \& Management. 1 sept 2019;13:39-50.

12. Senil N., Landel P-A. De la ressource territoriale à la ressource patrimoniale. In: Au cœur des territoires créatifs Proximités et ressources territoriales [Internet]. Presses Universitaires de Rennes. 2016. Disponible sur: https://halshs.archives-ouvertes.fr/halshs-

01387636/file/Ressources\%20patrimoniales\%20Vf.pdf

13. Berriane M., Michon G., éditeurs. Les terroirs au Sud, vers un nouveau modèle?: une expérience marocaine [Internet]. Marseille: IRD; 2016. Disponible sur: http://www.documentation.ird.fr/hor/fdi:010068624

14. Leniaud J-M. L'utopie française: essai sur le patrimoine. Mengès. Paris; 1992.

15. Amougou E. La question patrimoniale: de la" patrimonialisation" à l'examen des situations concrètes. Paris: Harmattan; 2004. 282 p.

16. Berriane M. Patrimoine et patrimonialisation au Maroc. Hesperis-Tamuda. 2010;XLV:11-7.

17. Davallon J. À propos des régimes de patrimonialisation: enjeux et questions. In Lisbonne; 2014.

18. François $\mathrm{H}$, Hirczak M, Senil N. Territoire et patrimoine: la co-construction d'une dynamique et de ses ressources. Revue d’Économie Régionale \& Urbaine. 2006;(5):683-700. 
19. Arab N. Pour une théorie du projet en urbanisme. Revue européenne des sciences sociales. 11 juin 2018; 56-1(1):219 - 40.

20. Pinson G. Chapitre 5 : Le projet urbain comme instrument d'action publique. In: Gouverner par les instruments [Internet]. Presses de Sciences Po; 2005. p. 199 - 233. Disponible sur: http://www.cairn.info/gouverner-par-les-instruments--9782724609492-page-199.htm

21. Ingallina P. Le projet urbain, une notion floue. In: Le projet urbain [Internet]. 3e éd. Presses Universitaires de France; 2008 . p. 7-13. (Que sais-je?). Disponible sur: http://www.cairn.info/le-projeturbain--9782130566106-page-7.htm

22. Lazarev G. Chapitre 5 - Promouvoir le développement des territoires ruraux. In: MediTERRA [Internet]. 2009. p. 183 à 210. Disponible sur: https://www.cairn.info.eressources.imist.ma/mediterra-2009-978272461109-page-183.htm

23. Landel P-A. L'exportation du «développement territorial» vers le Maghreb: du transfert à la capitalisation des expériences. L'Information géographique. 2011;Vol. 75(4):39 - 57.

24. Zouiten M. Gouvernance locale, partenariat organisé et décision communale. Critique économique. 2004; (13).

25. Pinson G. Gouverner par projet. Urbanisme et gouvernance des villes européennes, Paris, Presses de Sciences Po. 2009.

26. Vernières M. Le patrimoine: une ressource pour le développement. Techniques Financieres et Developpement. 2015; $n^{\circ} 118(1): 7$ - 20.

27. M'rini L., Alaoui A., Akerraz O., Skounti A. Éléments pour une vision patrimoine 2020. Ministère de la Culture; 2014

28.UNESCO. Comité du patrimoine mondial. État de conservation Rabat, capitale moderne et ville historique: un patrimoine en partage (Maroc) [Internet]. 2019 [cité 30 mai 2020]. Disponible sur: https://whc.unesco.org/fr/soc/3992/

29. Micoud A. Des patrimoines aux territoires durables. Ethnologie française. 2004;Vol. 34(1):13 - 22. 